\title{
Entornos virtuales como recurso didáctico en la asignatura Dibujo Técnico
}

\author{
Virtual environments as a teaching resource in the subject \\ Technical Drawing
}

\section{César Enrique Chirino López}

cchirino_21@gmail.com

Código ORCID: 0000-0002-2080-8184

\author{
Víctor Escalante \\ davictor12@hotmail.com \\ Código ORCID: 0000-0003-4786-5522
}

Ministerio del Poder Popular para la Educación, Venezuela

Recibido febrero 2020 / Arbitrado marzo 2020 / Publicado mayo 2020

\section{Resumen}

La finalidad de esta investigación fue analizar la importancia de las entornos virtuales como recurso didáctico en la asignatura Dibujo Técnico tercer año Escuela Técnica "Simón Bolívar"; fundamentada en la Teoría del Aprendizaje para la era digital de Stephen Downes y George Siemens pensada en el conectivismo y teoría de la tecnología de Cabrera. Estudio descriptivo apoyado a una investigación documental y de campo. Para esta investigación, la muestra estuvo representada por diecisiete estudiantes. Se empleó la técnica de la encuesta y como instrumento de recolección de datos se aplicó un cuestionario de respuestas dicotómicas. La validez y confiabilidad se determinó a través del coeficiente Kuder Richardson, quedando en 0,72 . El análisis de dato se procesó a través de interpretaciones estadísticos porcentuales, arrojando como resultado la necesidad e importancia de incorporar entornos virtuales como recursos didácticos complementarios en el proceso de enseñanza y aprendizaje a través de los medios tecnológicos.

\footnotetext{
Abstract

The purpose of this research is to analyze the importance of virtual environments as a didactic resource in the subject Technical Drawing third year Technical School "Simón Bolívar"; based on the Theory of Learning for the digital age of Stephen Downes and George Siemens thought on Cabrera's connectivism and technology theory. Descriptive study supported by documentary and field research. For this investigation, the sample was represented by seventeen students. The survey technique was used and a dichotomous response questionnaire was applied as a data collection instrument. The validity and reliability was determined through the Kuder Richardson coefficient, reaching 0,72. The data analysis was processed through percentage statistical interpretations, resulting in the need and importance of incorporating virtual environments as complementary didactic resources in the teaching and learning process through technological means.
}

Palabras clave:

Estrategia virtual; recurso didáctico; dibujo técnico

\section{Keywords:}

Virtual strategy; teaching resource; technical drawing 


\section{INTRODUCCIÓN}

$\mathrm{E}$ l modelo tradicional educativo tuvo mucho éxito durante más de un siglo, pero la sociedad ha cambiado tanto que entró en crisis. Las innovaciones se hicieron necesarias en el camino hacia la escuela de este nuevo siglo. La democracia moderna no podría concebirse sin la escuela. Principalmente porque fue un instrumento poderoso para formar una comunidad de valores en torno a una idea de nación, algo sumamente necesario para todos los países del mundo. $\mathrm{Y}$, en segundo lugar, porque al educar a la mayoría de la población como nunca antes había ocurrido, permitió el desarrollo de una ciudadanía despierta, lo que mejoró la calidad de la democracia, vale destacar:

La escuela pública ha producido una de las transformaciones más significativas de la humanidad. Hace un siglo y medio menos de la quinta parte de la población del mundo culminaba una educación básica, y hoy más del $80 \%$ lo hace. Esto es extraordinario. Haber logrado construir para los $7 \mathrm{mil}$ millones de habitantes del planeta una experiencia común en estas instituciones es algo sin precedente y constituye la revolución silenciosa más significativa que se ha alcanzado. (Reimers, 2017, párr. 2)

El sistema educativo ha pasado de ser un sistema rígido a uno flexible; el sistema educativo tradicional era totalmente rígido. Trataba a todos los estudiantes como si fueran iguales y pretendía que todos completaran sus estudios con los mismos conocimientos. Expresamente buscaba homogeneizar; esto es muy problemático en una sociedad que busca potenciar las individualidades y le exige a cada uno decidir qué hacer con su vida.

En la actualidad, las Tecnologías de la Información y Comunicación (TIC) posibilitan la creación de un nuevo espacio para las interacciones humanas posibilitando nuevos procesos de aprendizaje y comunicación del conocimiento; según Badia (2006, citado por Castañeda, 2010) "actualmente ya nadie pone en duda que el ordenador contribuye a proporcionar nuevos tipos de ayudas educativas o que puede cambiar la naturaleza de éstas, influyendo por consiguiente de manera diferencial en los procesos de aprendizaje de los estudiantes"( p.4). 
Hoy en día, los estudiantes construyen su propio conocimiento a través de la experiencia, según Silvio (2000) "El ordenador y todo el ambiente virtual que se ha creado es la llave para abrir nuevos horizontes para la enseñanza, el aprendizaje y la investigación" (p. 12). Por lo tanto, la educación en lo virtual (incluye educación presencial o no presencial) asegura a las nuevas generaciones nuevos conocimientos sin fronteras y el inicio de una cultura de comunicación digitalizada, que garantizará mejorar su calidad de vida. "La tecnología puede utilizarse para crear una comunidad digital en la que los estudiantes interactúen de manera que produzcan recursos para el aprendizaje". (Tobin, 1999, p. 18)

Esta utilidad de las TIC en la didáctica de las ciencias conlleva a nuevas formas de leer y escribir donde se requieren nuevos instrumentos y nuevas habilidades. La inclusión de nuevas formas de comunicación asíncrona (correo electrónico, tablones de anuncios, blogs, portales dinámicos) y síncronas (chats, videoconferencias) que se están realizando dentro de la sociedad, la cotidianidad y la misma ciencia son replicadas en lo pedagógico y didáctico, agrega Zárrate (2009, citado por Castañeda, 2010), que estas replicas manifiestan deficiencias a la hora de ejecutar las prácticas presenciales y se debe a que dichos ambientes no son ajustados a las necesidades de los estudiantes, porque se asume que "la tecnología ocupa un lugar subordinado y muy reducido en comparación con el reservado para la ciencia" (Cornejo,2006, citado por Castañeda, 2010), y dentro de la actividad docente.

La formación permanente de los docentes; sin duda alguna, los modelos educativos complejos, flexibles y personalizados demandan mucho más de los docentes que un esquema burocratizado en el que hay un programa predeterminado y el profesor se limita a seguirlo. Por eso resulta imperioso que quien esté al frente de una clase sea una persona preparada y cuente con una multiplicidad de recursos; es por ello que se necesitas maestros actualizados por las demandas que el contexto educativo.

La tecnología lleva varios años asistiendo a profesores y estudiantes en su trabajo diario. Los procesadores de texto, las calculadoras, las impresoras y los computadores se han utilizado desde hace décadas para las distintas actividades estudiantiles. Sin embargo, actualmente con internet y la tecnología móvil en auge se incorporan aún más elementos tecnológicos al entorno educativo. Pizarras interactivas, aulas virtuales y una variedad de 
recursos electrónicos para llevar a cabo investigaciones o realizar trabajos escolares son algunas de las formas en las que la tecnología digital se ha integrado con las escuelas y universidades. Al respecto Curtin (2017) director mundial de Educación Superior de Microsoft señala:

Es un momento de grandes oportunidades. Las universidades han usado tecnologías para ampliar el acceso a la educación. Aun así, las instituciones innovadoras de hoy están usando la nube inteligente para escalar experiencias de aprendizaje altamente personalizadas que más allá de la cobertura, mejoren la calidad. (párr. 5)

Por ende, el Dibujo técnico desde esta óptica puede adaptarse a EVA implementándose una metodología de apoyo virtual como complemento a la modalidad presencial de la asignatura, consistente en el uso de las diversas plataformas tecnológicas y conectadas a ellas herramientas de interacción de la web 2.0 que le permitan al estudiante desarrollar con ellas talleres en los que intervendrán los estudiantes y el docente, donde se requiere un abordaje dinámico, práctico y reflexivo. Incluye la realización de ejercicios o tareas individuales que den cuenta del proceso de aprendizaje alcanzado, los cuales serán trabajados y calificados en la virtualidad.

En Venezuela es considerada la educación como un derecho fundamental, por ello está contemplado en Ley Orgánica de Educación (2009) en su artículo 14 precisa:

La educación es un derecho humano y un deber social fundamental concebida como un proceso de formación integral, gratuita, laica, inclusiva y de calidad, permanente, continua e interactiva, promueve la construcción social del conocimiento, la valoración ética y social del trabajo, y la integralidad y preeminencia de los derechos humanos, la formación de nuevos republicanos y republicanas para la participación activa, consciente y solidaria en los procesos de transformación individual y social. La educación regulada por esta Ley se fundamenta en la doctrina de nuestro Libertador Simón Bolívar, en la doctrina de Simón Rodríguez, en el humanismo social y está abierta a todas las corrientes del pensamiento. (pp. 16-17) 
Se considera entonces que la educación es vista como el eje central de formación de toda persona, porque a través de ella se promueven valores éticos y morales y, sobre todo, como un derecho fundamental de todos y todas los venezolanos y venezolanas, lo cual busca fortalecer los proceso educativo y social a través de acciones constructivas donde se promuevan las medidas efectivas.

Considerando pues que hoy por hoy la gran connotación que tiene la educación virtual, también denominada educación en línea o educación a distancia, la cual se refiere al desarrollo de programas de formación que tienen cono escenario de enseñanza y aprendizaje el ciberespacio apoyándose en las TIC; ya que der un docente online no es solo cambiar una clase presencial por una online, enviar documentos electrónicos o crear foros para mostrar información adicional a la clase; un tutor virtual debe reinventarse para aprovechar las herramientas que ofrece la tecnología, y, en muchos casos, debe diseñar nuevas tácticas que permitan un mejor aprendizaje en un medio virtual, aunado a eso, debe ejercer el rol de promotor y mediador de este proceso.

No obstante, se en tiende que este tipo de formación requiere algunos complementos o estrategias online adicionales que ayuden aún más al alumnado a recibir una formación de calidad. Y esta opción no está sólo en el profesor, sino en las características de la plataforma online que sustenta su enseñanza, que debe contar con las herramientas necesarias para que éste logre sus objetivos educacionales.

En función a esto, existen investigaciones en este ámbito las cuales inciden significativamente en el uso de los entornos virtuales en la enseñanza, por ejemplo: Jiménez y Reyes (2017) en su investigación titulada aula virtual como recurso didáctico en la asignatura dibujo en los estudiantes de la mención educación para el trabajo de la FaCE-UC. Cuya finalidad fue proponer un aula virtual como recurso didáctico en la asignatura dibujo en los estudiantes de la mención educación para el trabajo de la FaCE-UC, a fin de solventar hechos evidentes como lo es la falta de recursos didácticos, desplazamiento de herramientas virtuales las cuales ayudan en la construcción del conocimiento.

Por su parte Agudo (2016) en su trabajo titulado Propuesta de aula virtual como estrategia de aprendizaje para la asignatura Dibujo Técnico, Unidad Educativa "Mercedes Izaguirre de Corro", esta investigación tuvo como fin proponer el uso de un aula virtual como 
aprendizaje para la asignatura Dibujo técnico en estudiante de segundo año de educación media, apoyándose además de los distintos recursos tecnológicos disponibles en la unidad educativa. En la misma concluye que el uso de las TIC como herramienta de apoyo en las actividades educativas, en las modalidades presencial y virtual no sólo requiere del conocimiento necesario por parte del docente, sino de su habilidad para adaptarlas a los cambios que experimentan la sociedad y el contexto educativo. A su vez, se debe tener en cuenta que el contexto social es un factor determinante del éxito o fracaso de las TIC.

En el caso de los estudiantes del tercer año, del Escuela Técnica "Simón Bolívar", debido a la grave situación que atraviesa Venezuela, se observa la carencia de los recursos materiales para la realización de las actividades prácticas en el taller de dibujo, como: lápices, borrador, sacapuntas, juego de escuadras, compás y block de dibujo, entre otros lo que origina debilidades en la asignatura Dibujo técnico, los estudiantes no hacen uso de las diferentes herramientas como factor primordial en mundo tecnológico y en el campo educativo las cuales le permitan estar a la vanguardia en los avances y gestión de conocimiento de acuerdo a su nivel.

En la actualidad se implementa notablemente la educación a distancia, que viene a ser un complemento de los encuentros presenciales de clases, donde los espacios virtuales juegan un papel fundamental. Es decir es necesario innovar en cuanto a las estrategias, pues la enseñanza se concibe como un proceso interactivo de acción pedagógica, mediante el cual se diseñan estrategias y se crean situaciones de aprendizaje acordes con los conocimientos previos, intereses, necesidades, gustos y preferencias de los aprendices. Desde la perspectiva constructivista la enseñanza, según Figueroa (2009).

Puede ser considerada como una tarea a través de la cual (alguien), en este el caso el docente, a través de estrategias de enseñanza, intenta ayudar a uno o más estudiantes a construir la comprensión, competencias y actitudes referidas a la realidad. (p.39)

En dicha institución educativa, los actores del proceso de enseñanza y aprendizaje no hacen uso de las herramientas 
tecnológicas que tienen a su disposición dentro del plantel, como, los talleres de informática por falta de conexión a internet y el mismo estado operativo de las máquinas; las cuales requieren mantenimiento constante y sobre todo de un personal responsable de velar por la preservación de los equipos.

Por lo antes expuesto, se hace necesaria recuperación de estos recursos tecnológicos para el aprovechamiento los entornos virtuales, lo cual hará más efectivo el aprendizaje y el alcance de las competencias de la asignatura Dibujo técnico. En función a todo lo expresado se tiene como objetivo general analizar la importancia de los entornos virtuales como recurso didáctico en la asignatura Dibujo técnico tercer año en la Escuela Técnica "Simón Bolívar", Municipio Naguanagua, Estado Carabobo.

Es importante señalar, para el logro del mismo fue necesario en primer lugar diagnosticar el conocimiento que poseen los docentes acerca de los entornos virtuales como recurso didáctico en la asignatura Dibujo técnico tercer año. En segundo lugar, describir las teorías entornos virtuales y recurso didáctico; para así interpretar los resultados evidenciados por los informantes clave. Ahora bien, es necesario evidenciar la practicidad del uso de la tecnología en pro de la educación ha apoyado en la Tecnología de Información y Comunicación (TIC) como herramienta y recurso didáctico, a través diversas estrategias de enseñanza que podamos como docentes poner en práctica en la modalidad virtual, buscando estimular en interés en los alumnos para lograr un mejor rendimiento y a su vez que el estudiante se interese cada vez en una herramienta innovadora que didácticamente los lleve a estar inmerso en los cambios tecnológicos de nuestra sociedad actual.

Las TIC promueve nuevos enfoques y nuevas soluciones a los problemas, provoca intelectualmente a su gente estimulándoles a hacerse preguntas, cuestionando los modos habituales de hacer las cosas, permitiendo que tengan errores. En tal sentido, el docente en su función laboral requiere promover en sus seguidores (estudiantes) la estimulación intelectual, ya que su aspecto esencial reside en proponer nuevo enfoque para viejos problemas; es decir, el educador debe hacer énfasis en la inteligencia y la racionalidad para la solución de problemas que se le puedan. Se trata de estimular la actuación, animándolos a generar nuevas ideas y a tomar responsabilidades, proporcionando así nuevos enfoques a los 
problemas, facilitando las cosas, no imponiéndolas. Según Zea (2001, citado por Castañeda, 2010) plantea:

La tecnología de la información no es un fenómeno tan nuevo como pretenden algunos. El proceso de construir artefactos (en el sentido más amplio del término) que favorezcan la preservación y circulación de información, con el fin de que podamos transformarla en conocimiento útil, ha sido una actividad constante desde los inicios de la palabra escrita. Lo novedoso hoy, es el hecho de haber puesto juntos numerosos recursos tecnológicos que generan una sinergia comunicativa sin precedentes: palabra escrita; registros orales y visuales; dispositivos masivos de almacenaje con capacidades de ordenar, organizar y transformar información; dispositivos potentes de transmisión y comunicación; disponibilidad casi universal de estos recursos; desaparición los condicionantes de tiempo y espacio. (p.6)

En el otro orden de ideas, este trabajo de investigación se justifica porque a nivel tecnológico, está conformado por dos áreas, el área la tecnología y los recursos didácticos, el primero fundamenta el uso de las diversas plataformas de las que se puede hacer uso para la difusión de las estrategias y lo segundo es, que se requiere de la utilización de medios didácticos para su mejor comprensión.

A nivel teórico esta investigación se justifica porque la aplicación de las estrategias a través de los entornos virtuales conlleva estimular el interés y a su vez a la obtención de conocimientos de forma práctica, sino también el aprovechamiento de las herramientas tecnológicas de fácil acceso a la que se está en presencia hoy día y su uso radica en aprender de forma didáctica. Y metodológicamente se justificó porque las estrategias son pilares fundamentales para que se lleve a cabo el proceso de enseñanza, adicionalmente a esto vamos aprovechar eficientemente los avances tecnológicos como herramienta para las estrategias; tal como lo precisa Briceño (2011) señala: “(...)el lugar, donde se imparte enseñanza tradicional conocido como aula de clases, puede ser sinónimo de ambiente, escenario o espacio de aprendizaje, puesto que, allí es donde se 
produce un acercamiento vivencial de los actores del proceso (...)" (p.55)

En este sentido, los estudiantes del tercer año de Escuela Técnica "Simón Bolívar", en la asignatura Dibujo Técnico, tendrán una herramienta más a través de las estrategias virtuales empleadas como recurso didáctico en la construcción de nuevos conocimientos; asimismo podrán llevar a cabo este proceso de manera novedosa y estando a la vanguardia con los avances y nuevas formas de gestión de conocimientos.

Asimismo es importante destacar, esta investigación posee relevancia ya que existen diversas herramientas tecnológicas a disposición de la educación; tales como: blogs, la cual es una herramienta muy rica en cuanto a funcionalidades. Puede actualizarse con mucha frecuencia y la información aparece categorizada según el interés de su creador. Tiene un estilo informal o conversacional y posibilita el intercambio de opiniones entre alumnos y docentes. Como facilitador online, puede utilizarlo para colocar información adicional o páginas de interés, ahondar en temas que requieren mayor extensión o son difíciles de entender, tareas o asignaciones, imágenes o vídeos de cualquier tipo, etc.

También se cuentan con Comunidades o Foros. Los cuales se han convertido en el mayor recurso de contacto con el alumnado de las empresas de E-learning. Los chat, los cuales permiten un contacto más rápido sincrónico entre los participantes y facilitador. Los $\mathrm{M}$ Learning, donde el aprendizaje móvil está en continuo crecimiento y es una estrategia de enseñanza que no se debe olvidar. El acceso a contenidos es rápido y facilita la colaboración entre los participantes. Vale destacar que aún siguen emergiendo nuevas herramientas las cuales están a disposición del docente y el estudiante.

\section{MÉTODO}

$\mathrm{E}$ n este apartado se encuentran las consideraciones generales de la investigación en donde se expone una breve explicación de cómo fue abordado la obtención de los datos, su relación con el objeto de estudio, las posibles delimitaciones respuestas a las interrogantes planteadas en el trabajo investigativo. 
Al respecto Arias (2006), expresa que "la metodología del proyecto incluye el o tipos de investigación, las técnicas y los procedimientos que serán utilizados para llevar a cabo la indagación. Es como se realiza el estudio para responder al problema planteado" (p.19). Por tal motivo, para efectos de esta investigación centrada en analizar la importancia de los entornos virtuales como recurso didáctico en la asignatura Dibujo Técnico tercer año en la Escuela Técnica "Simón Bolívar", se abordó desde un paradigma positivista, con enfoque cuantitativo donde el diseño y tipo fue de carácter no experimental de campo ya que no se van a manipular las variables; sólo se irá al lugar donde ocurren los hechos a fin de recoger información. A su vez este estudio fue de corte descriptivo ya que consiste en la caracterización de un hecho, fenómeno o grupo, con el fin de establecer su estructura o comportamiento.

Este tipo de investigación se asocia con el diagnóstico. El método se basa en la indagación, observación, el registro y la definición. "Los estudios descriptivos miden de forma independiente las variables y aun cuando no se formulen hipótesis, tales variables aparecen enunciadas en los objetivos de investigación" (Arias, 2006, p.25).

Ahora bien, la población asumida para esta investigación estuvo conformada por los estudiantes de la Escuela Técnica "Simón Bolívar"; al respecto señala Balestrini (2006) “(...) estadísticamente hablando, por Población se entiende un conjunto finito o infinito de personas, casos o elementos que presentan características comunes" (p.137). En cuanto a la muestra intencional, estuvo conformada por diecisiete estudiantes del tercer año sección " $\mathrm{D}$ " pertenecientes a la mención construcción civil de los cuales eran cursantes de la asignatura Dibujo Técnico en la institución.

Como técnicas e instrumentos seleccionados para este estudio, se tiene: observación directa, entrevista y el instrumento aplicado fue una encuesta la cual estuvo constituida por once ítems, con alternativas de respuesta Sí y No. Posterior a la elaboración del cuestionario se procedió a la validez de expertos quienes revisaron y evaluaron el contenido de este, al respecto Bernal (2010) dice que "un instrumento es válido cuando mide aquello para lo cual está destinado" (p. 214), razón por la cual, el mismo fue sometido a prueba de validez de juicio de expertos en el área.

Esta validación consistió en la revisión técnica del cuestionario por tres (03) especialistas en el área adscritos al Departamento de Administración y Planeamiento Educativo de la Facultad de Ciencias 
de la Educación Universidad de Carabobo; quienes emitirán juicio valorativo en torno al instrumento en atención a la consistencia interna, validez de contenido, redacción y coherencia. "Se justifica por el requerimiento metodológico de confrontamiento de criterios técnico, epistémicos y disciplinares del ámbito de estudio, congruencia y pertinencia teórica que debe poseer todo instrumento con carácter científico" (Sandín, 2003, p. 185).

A su vez se realizó el estudio de confiabilidad, donde se procedió a la aplicación de una prueba piloto a una muestra con características similares a la de la investigación, a partir de los resultados obtenidos se calculó la misma. Vale destacar, ésta se refiere al:

Nivel de exactitud y consistencia de los resultados obtenidos al aplicar el instrumento por segunda vez en condiciones tan parecidas como sea posible, genere resultado similar a la vez anterior, a nivel de exactitud y consistencia de los resultados obtenidos al aplicar el instrumento por segunda vez en condiciones tan parecidas como sea posible. (Hurtado y Toro, 2009, p.45)

Para la medición de los Ítem presentados se aplicó el coeficiente de Kuder Richardson $\left(\mathrm{KR}_{20}\right)$, ya que permite obtener la confiabilidad a partir de los datos obtenidos. Como conclusión se obtuvo una confiabilidad de 0,72 para este coeficiente lo que quiere decir que se obtuvo una confiabilidad Alta.

\section{RESULTADOS Y DISCUSIÓN}

$\mathrm{E}$ n esta etapa, la investigación se desarrolló a partir de los datos cuantitativos, tomados de la Escuela Técnica "Simón Bolívar", ubicado en el municipio Naguanagua del estado Carabobo. A fin de profundizar la información fue necesario agruparla en un cuadro metodológico para determinar la masa de datos por dimensiones e indicadores, facilitando así el proceso de análisis e interpretación de los resultados.

Para lograr el razonamiento se hizo una representación porcentual de las respuestas, agregando en cada gráfica su respectiva interpretación, apoyada siempre en los datos obtenidos, y de esta 
manera darle el carácter científico requerido por la investigación en contraste con los fundamentos teóricos de la misma. Cabe destacar que se procedió a representar los ítems por dimensiones en atención a las variables planteadas para esta investigación las cuales son entornos virtuales definido como "un espacio educativo alojado en la web, conformado por un conjunto de herramientas informáticas que posibilitan la interacción didáctica" Salinas (2011, p.24). Y la otra variable recurso didáctico, definida como cualquier material que se ha elaborado con la intención de facilitar al docente su función y a su vez la del alumno. No olvidemos que los recursos didácticos deben utilizarse en un contexto educativo. Todo esto con la finalidad de establecer conclusiones en forma puntual, partiendo de los indicadores propuestos en cada una de estas.

A continuación se presentan cinco ítems más relevantes presentes en el instrumento usado con la finalidad de obtener información precisa. En cuanto a la primera variable, se tiene el ítem 1: ¿Te gustaría el uso de entornos virtuales en el dibujo técnico? En este caso los informantes indicaron que 88 porciento le gustaría el uso de entornos virtuales en el dibujo técnico, mientras que el 12 por ciento indicó que no le gustaría su inclusión. Esto se puede apreciar en el Grafico 1.

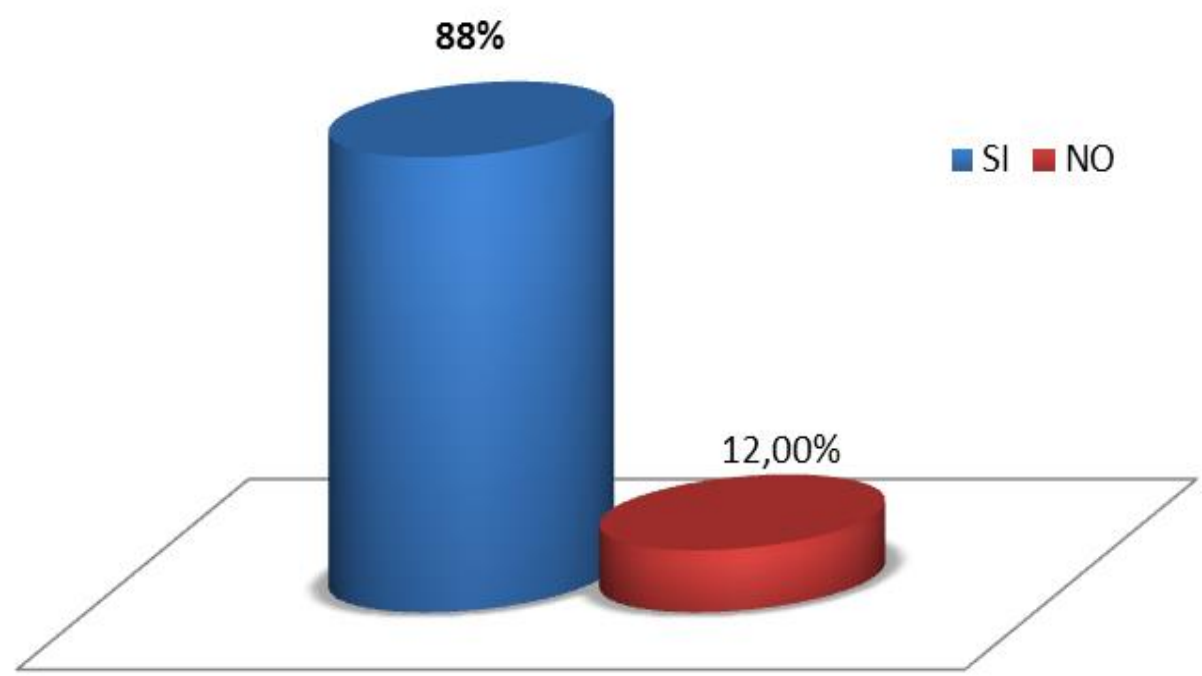

Gráfico 1. Inclusión de los entornos virtuales en al aula. (Fuente: Chirinos y Escalante, 2020)

En los resultados obtenidos se puede evidenciar la necesidad que tienen los estudiantes de contar con otros escenarios didácticos en su institucion educativa; de hecho, los estudiantes ven sumamente 
importante la inclusión de recursos virtuales en el ámbito escolar espáticamente en la materia Dibujo Técnico manifestando que es de gran ventaja a la hora de iniciar temas complejos referentes a la materia. Tal como lo afirma Crespo (2008):

Más allá de estas definiciones, es necesario reflexionar en qué medida las TIC favorecen o no a las poblaciones, y si éstas están llegando de manera equitativa; por una parte tiene impacto en la cotidianidad de las personas pero a su vez crea una mayor exclusión, bien sea por causas económicas, sociales, cognitivas, políticas, entre otras. El mayor reto es crear las condiciones necesarias para reducir las diversas brechas, a fin de que los menos favorecidos puedan sacar provecho de las TIC. (p. 27)

Y en efecto, más que un reto es un desafío para los docentes lograr la incorporación de los entornos virtuales en las asignaturas que facilita; existe resistencia pues no es fácil romper paradigmas ya instaurados. En este sentido, se tiene el ítem 2: ¿Los entornos virtuales son una ventaja en el dibujo técnico?

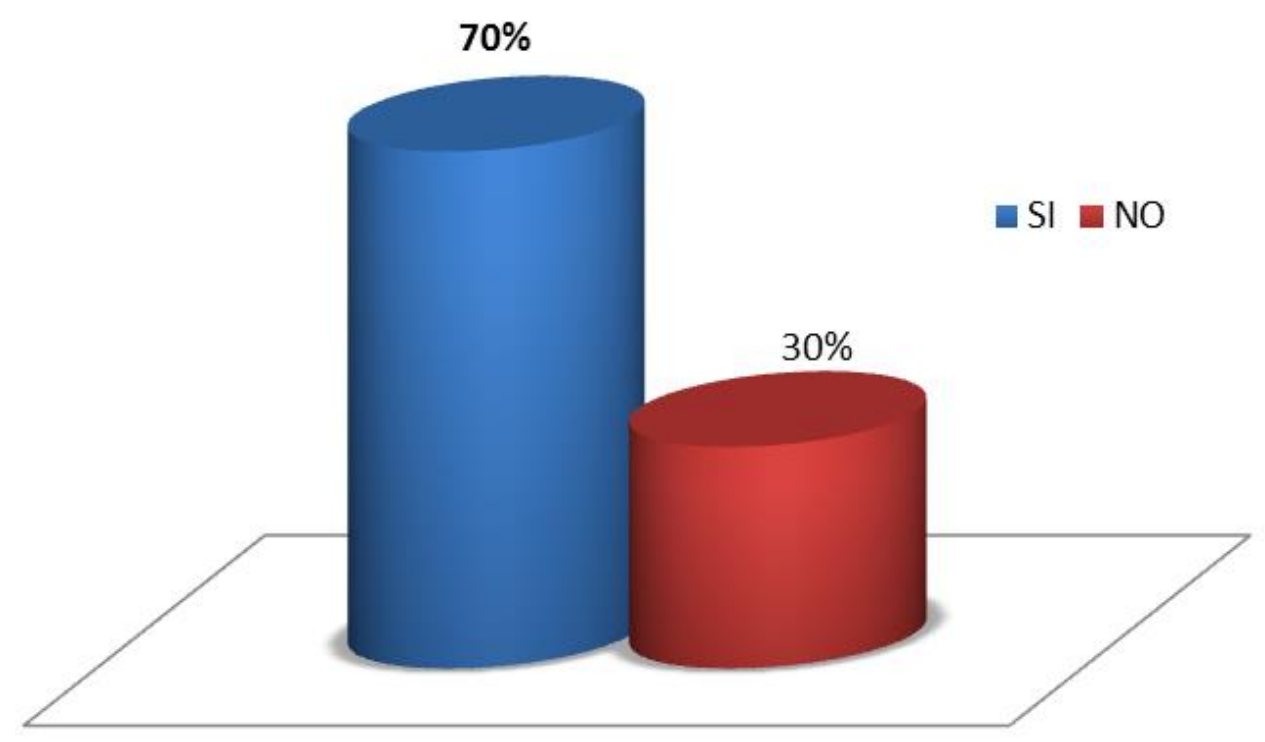

Gráfico 2. Ventajas de los entornos virtuales. (Fuente: Chirinos y Escalante, 2020)

En el Gráfico 2 se puede apreciar que el 70 por ciento de los encuestados afirman que los entornos virtuales son una ventaja en el dibujo técnico, mientras que el $30 \%$ índico que estos no sería un 
cambio relevante; es decir, los resultados obtenidos indican que los estudiantes ven sumamente importante la inclusión de recursos virtuales en el ámbito escolar espáticamente en la materia dibujo técnico manifestando que es de gran ventaja a la hora de iniciar temas complejos referentes a la materia.

Por lo que las necesidades detectadas están en sintonía con lo precisado por Felicié (2006) en cuanto a las ventajas, pues las TIC: "Contribuyen a potenciar el aprendizaje y la educación a distancia, proveyendo a la población nuevos medios para la educación en todas las etapas de la vida" (p.18). Mediante las estadísticas se puede notar la gran influencia de los entornos virtuales en el medio educativo donde la mayoría ha tenido contacto o conoce su funcionamiento y reconoce algunos métodos de aprendizaje mediante el mismo. Ahora bien, en cuanto a la variable recurso didáctico, en especial la dimensión funciones se encuentra el ítem 6: ¿Usas buscadores virtuales para obtener información?

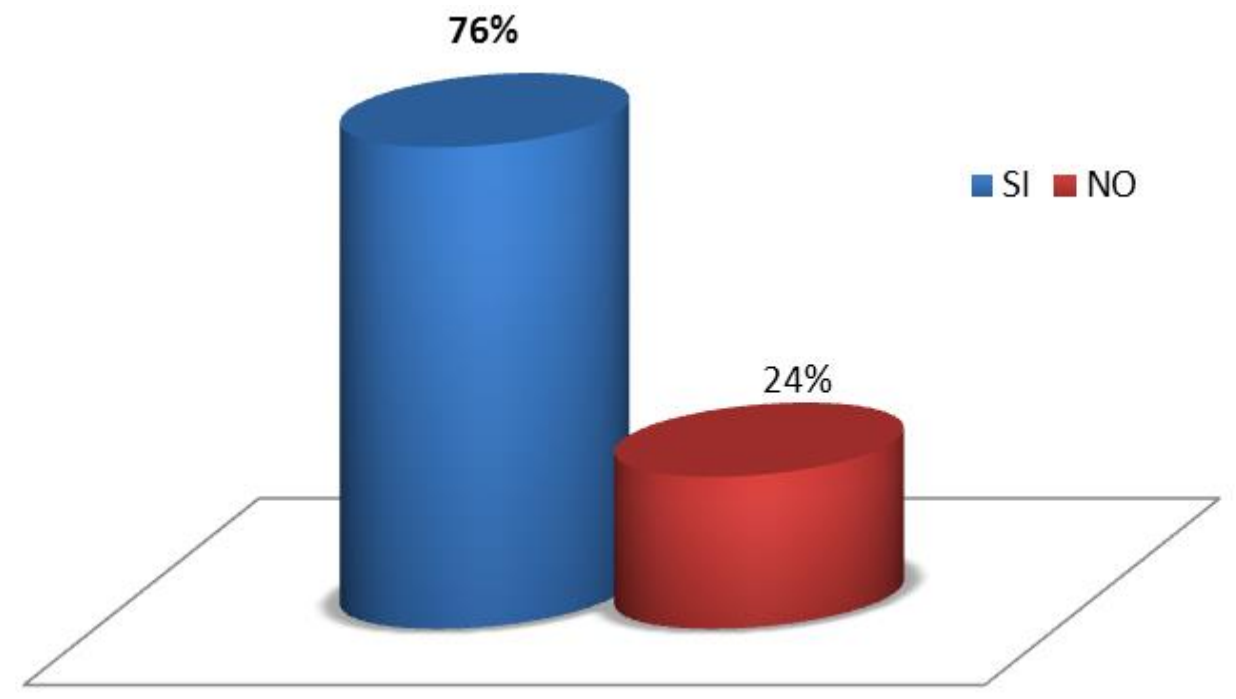

Gráfico 3. Uso de los buscadores en entornos virtuales. (Fuente: Chirinos y Escalante, 2020)

En este sentido se puede apreciar en el Gráfico 3 que el 76\% de los encuestados han usado buscadores virtuales para obtener información, mientras que el $24 \%$ señalaron no haber utilizado buscadores. Quedando evidenciado que la mayoría de los participantes consideran útiles los buscadores al momento de obtener información, conectarse con el mundo a través de las redes. 
Por ello la importancia de lo precisado por García y Tejedor (2010, citados por Bravo 2017):

La Informatización de la sociedad, la cual ha hecho posible poner en contacto grupos sociales de todo el mundo a un mismo tiempo, superando así las distancias y la dispersión geográfica; la Globalización concebida como un proceso de integración de las distintas actividades humanas (sobre todo las que tienen que ver con las redes de información y corrientes culturales); y las Nuevas Tecnologías. (p. 16)

Teniendo en cuenta estas consideraciones, se hace entonces necesario asumir una postura crítica ante los nuevos desafíos que se presentan en el ámbito educativo en cuanto a la TIC respecta. Ya que hoy en día el uso de los las ha tenido un crecimiento elevado.

Se puede evidenciar por ejemplo en el caso educativo a través del ítem 7: ¿Utilizas con frecuencia entornos virtuales en el ámbito educativo? En el Grafico 4 se puede apreciar, el $82 \%$ de los encuestados afirma utilizar con frecuencia entornos virtuales en el ámbito educativo a la hora de hacer tareas, 18\% aclara nunca haber usado entornos virtuales.

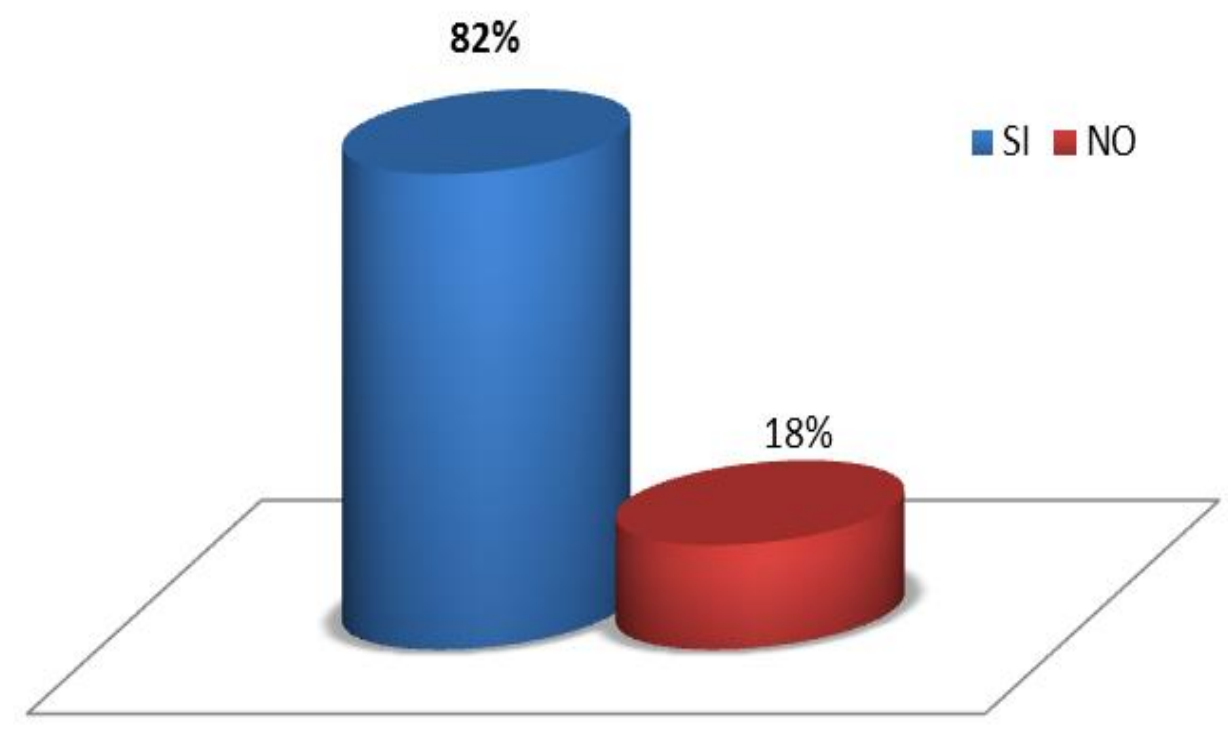

Gráfico 4. Entornos virtuales en el ámbito educativo. (Fuente: Chirinos y Escalante, 2020) 
Estos resultados permiten dilucidar el impacto de las TIC en el ámbito educativo, pues queda claro que la mayoría de los estudiantes ya maneja este recurso; por lo cual es viable que en la institución educativa implementen los entornos virtuales como recurso didáctico en el asignatura Dibujo técnico; sin perder de vista la postura de George Siemens (2010, citado por Bravo, 2017), el cual hace referencia:

El Conectivismo como teoría presenta un modelo de aprendizaje que refleja una sociedad en la que el aprendizaje ya no es una actividad individual. Ahora se trata de reconocer el hecho de que los modos de aprender y su función se alteran cuando se utilizan nuevas herramientas. (p. 17)

Seguidamente, se tiene el ítem 9: ¿Es práctico el uso de entornos virtuales en el dibujo técnico?; el 70\% de los encuestados expresó que es práctico el uso de los mismos en el dibujo técnico, por otro lado, en $30 \%$ no nota la relevancia; así se puede apreciar en el Gráfico 5.

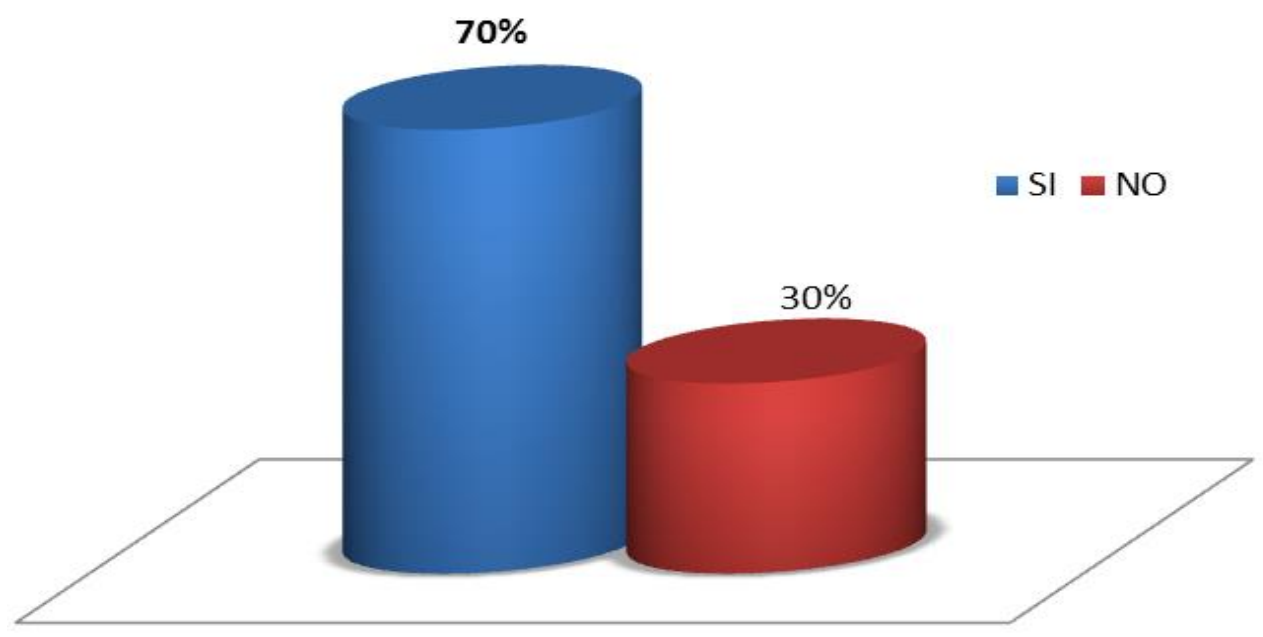

Gráfico 5. Practicidad de los entornos virtuales. (Fuente: Chirinos y Escalante, 2020)

De esta manera se concluye que los estudiantes de tercer año sección " $\mathrm{D}$ " pertenecientes a la mención construcción civil de la Escuela Técnica "Simón Bolívar" ven necesario el uso de entornos virtuales en el área de dibujo técnico por las grandes ventajas que representa el uso de este recurso, validando así lo conceptualizado Siemens (2004), señala: “(...) aprendemos haciendo conexiones con 
otras personas, conceptos e ideas. Esas conexiones pueden ser de diversos tipos: de tipo social, "cara a cara", o de tecnológicas". (p.28)

Para ello se amerita de la preparación no sólo del personal docente sino de todos los actores involucrados en el proceso de enseñanza y aprendizaje, para optimizar el uso y el mejor aprovechamiento de las herramientas que ofrecen las TIC y que a través de ellas se puedan lograr los objetivos que persigue el actual Sistema Educativo.

\section{CONCLUSIONES}

$\mathrm{E}$ l objetivo de este trabajo de investigación centrado en analizar la importancia de los entornos virtuales como recurso didáctico en la asignatura Dibujo Técnico, surgió a fin de optimizar el aprovechamiento de las herramientas tecnológicas como interfaz de aprendizaje. Debido a la situación país, además de ser importante el uso se los entornos virtuales de aprendizaje, ya que la educación debe estar a la vanguardia de los avances tecnológicos, se hace necesario para subsanar los altos costos de los instrumentos físicos esenciales para la realización de los talleres y clases de dibujo técnico en esta institución.

El proceso de enseñanza y aprendizaje en esta asignatura estaría reforzado ya que la carencia de los recursos físicos no sería impedimento para que proceso de enseñanza se lleve a cabo y se logren los objetivos propuestos; la virtualidad sería un aditivo positivo para tal fin. En atención a los resultados obtenido en cuanto a la confiabilidad del instrumento aplicado, se concluye que es necesario el uso de los entornos virtuales para la asignatura de Dibujo técnico en dicha institución.

Debido a las diferentes estrategias que se pueden llevar a cabo a través del apoyo que representan los entornos virtuales en la educación y en al caso específico de la asignatura de Dibujo técnico, el docente puede cumplir a cabalidad con el plan de clase y el plan de evaluación, ya que la flexibilidad característica de los entornos virtuales así lo permiten. Hoy día se vive en la era tecnológica y la educación uno de los pilares fundamentales para el desarrollo de la sociedad, por ende no debe estar aislada de los avances de la tecnología; por lo tanto nosotros como docentes debemos aprovechar el gusto de los nuevos estudiantes por la tecnología, para así sacar el 
mejor provecho a las estrategias de aprendizaje desde los entornos virtuales.

De allí, se recomienda reacondicionar el taller y los equipos informáticos con los que cuenta el plantel para aprovechar al máximo su uso; además ubicar dentro del plan de lapso horas destinadas al uso de los entornos virtuales, designar un personal encargado del taller de informática del plantel que esté al pendiente del cuidado y mantenimiento del mismo y sus equipos

Para el logro de todo esto, es importante conseguir la disposición del personal, por lo cual se debe incentivar a los docentes para su capacitación y actualización en el uso los entornos virtuales. En el caso de los estudiantes, razón de ser de la educación, es necesario orientar y asesorar de forma constante en el uso adecuado de estos entornos; asimismo, conocer sus expectativas, necesidades, dificultades y experiencia en el uso de la tecnología para usar las estrategias adecuadas en los entornos virtuales; puesto que actualmente se debe aprovechar los avances tecnológicos en pro de cubrir las necesidades que se presentan en la cotidianidad de nuestro entorno social y educativo, con el objeto de beneficiarse de forma productiva para nuestro desarrollo cognitivo.

\section{REFERENCIAS}

Arias, F. (2006). El Proyecto de Investigación. Guía para su elaboración. ( $3^{\mathrm{a}}$ ed.) Caracas: Texto

Agudo, T. (2016). Propuesta de Aula Virtual como Estrategia de Aprendizaje para la Asignatura Dibujo Técnico Unidad Educativa Mercedes Izaguirre de Corro. Recuperado de http://mriuc.bc.uc.edu.ve/handle/123456789/3121

Aparici, R. y García, A. (1988). El material didáctico de la UNED. Madrid: ICE-UNED

Balestrini, M. (2010). Cómo se elabora el proyecto de investigación. $7^{\mathrm{a}}$. (ed.): Consultores Asociados

Bernal, C. (2010). Metodología de la investigación: para administración, economía, humanidades y ciencias sociales ( $3^{\underline{a}}$ ed.): Colombia: Pearson Educación

Bravo, K. (2017). Aprendizaje de ondas y óptica desde la visión del conectivismo en el subsistema de educación universitaria. (Tesis de Maestría) Recuperado de http://mriuc.bc.uc.edu.ve/handle/ $123456789 / 4404$ 
Briceño, T. (2011). El uso del error en el aprendizaje. Una posible construcción pedagógica argumentativa. Venezuela: Corporación ASM, C.A

Castañeda, A. (2010). La importancia del mundo virtual en la enseñanza y aprendizaje AVA para el contenido de Genética. Biografía: Escritos sobre la Biología y su Enseñanza 3 (5). Recuperado de https://doi.org/10.17227/20271034.vol.3 num.5bio-grafia116.137

Crespo, E. (2008). Guía para el análisis del impacto de las Tecnologías de la Información. España: Universidad Politécnica de Madrid. Recuperado de http://oa.upm.es/1045/1/pfc_enrique crespo molera.pdf

Curtin, R. (2017). Líderes mundiales en tecnología hablan sobre "La era digital en la educación. Recuperado de https://www.technoymas.com/lideres-mundiales-en-tecnologiahablan-sobre-la-importancia-de-la-era-digital-en-la-educacion/

Felicié, A. (2006). Biblioteca pública, sociedad de la información y brecha digital. Buenos Aires: Alfagrama. Recuperado de http://hdl.handle.net/123456789/2576

Figueroa, L. (2009) El constructivismo y el aprendizaje de los estudiantes. Recuperado de https://www.redalyc.org/pdf/373/ 37319199005.pdf

Hurtado, L., y Toro, J. (2009). Paradigmas y Métodos de Investigación en tiempos de Cambio. Valencia, Carabobo, Venezuela

Jiménez, G. y Reyes E. (2017). Aula Virtual como Recurso Didáctico en la asignatura dibujo en los estudiantes de la Mención Educación para el Trabajo de la FACE-UC. (Trabajo Especial de Grado). Recuperado de http://mriuc.bc.uc.edu.ve/bitstream/ handle/123456789/4441/15627.pdf?sequence $=3$

Ley Orgánica de Educación. Gaceta Oficial de la República de Venezuela, 5.929. (Extraordinario), Agosto 15, 2009

Reimers, F. (2017). Los 3 cambios más disruptivos que están transformando la educación mundial. Recuperado de http://otrasvoceseneducacion.org/archivos/208744

Salinas, M. (2011). Significado de entornos virtuales de aprendizaje en docentes. Recuperado de http://otrasvoceseneducacion.org/ archivos/100645

Sandín, E. (2003). Investigación cualitativa en educación. Fundamentos y tradiciones. Madrid, España: Mc Graw Hill Interamericana de España

Siemens, G. (2004). Conectivismo: Una teoría de aprendizaje para la era digital. Recuperado de https://pdfs.semanticscholar.org 105f1/adee187323d66beab226058b23a7416c3517.pdf 
Siemens, G. (2010). Conociendo el conocimiento. Recuperado de http://www.nodosele.com/editorial

Silvio, J. (2000). La virtualización de la universidad, ¿cómo transformar la educación superior con la tecnología? Caracas: IESALC/UNESCO

Tobin, L. (1999). La tecnología puede utilizarse para crear una comunidad digital en la que los estudiantes interactúen de manera que produzcan recursos para el aprendizaje. Recuperado de https://www.academia.edu/29516759/ambiente_virtual_de aprendizaje_en_gen\%c3\%a9tica 